\title{
Conductas disruptivas desde la óptica del docente: validación de una escala
}

\author{
Marina Álvarez Hernández ${ }^{1 *}$, Pilar Castro Pañeda ${ }^{1}$, Carmen González-González de Mesa² \\ Eva Álvarez Martino ${ }^{3}$ y Marián Ángel Campo Mon ${ }^{1}$

\footnotetext{
1 Universidad de Oviedo, Facultad de Formación del Profesorado y Educación, Departamento de Psicología. (España).

${ }^{2}$ Universidad de Oviedo, Facultad de Formación del Profesorado y Educación, Departamento de Ciencias de la Educación. (España).

${ }^{3}$ Universidad de Oviedo, Facultad de Formación del Profesorado y Educación, Departamento de Filosofía. (España).
}

\begin{abstract}
Resumen: En los últimos años han aumentado significativamente las dificultades para impartir clases, debido a lo que se conoce como disrupción en el aula. Prueba de ello son las numerosas publicaciones recientes en torno al tema, así como la frecuencia con la que dicha cuestión aparece en los medios de comunicación. El objetivo principal de esta investigación consiste en validar una escala con el propósito de conocer objetivamente la opinión de los profesionales de la educación sobre las conductas disruptivas en el aula. Teniendo en cuenta literatura previa y utilizando un panel de expertos, se desarrolló una nueva escala de 15 ítems. Se aplicó a una muestra de 346 profesionales, con una media de edad de 43 años.

Se llevó a cabo un análisis factorial exploratorio, un análisis de componentes principales y un análisis de correlaciones entre factores. Dichos análisis han permitido comprobar que la estructura factorial de las escalas converge con los factores previstos y reafirma su peso y nivel de confianza. Todo ello permite concluir que la escala es válida para medir la óptica del profesorado respecto a la cuestión objeto de estudio. Los datos indican un óptimo ajuste de la estructura tridimensional para los ítems de la escala.

Palabras clave: escala; Likert; conductas disruptivas; convivencia escolar; educación; validez; fiabilidad; análisis factorial exploratorio.
\end{abstract}

Title: Disruptive behaviours from teacher's perception: validating a scale. Abstract: In recent years difficulties in the field of teaching have increased considerably, due to what it is known as disruptive classroom behaviour. Proof of this can be seen in the fact that this topic is the focus of numerous recent studies and also in the fact that this question appears frequently in the media. The main purpose of this study is to validate a scale in order to know objectively the views of educational professionals about disruptive classroom behaviours. Taking into account previous literature and working with a panel of experts, a new scale of 15 items was developed. The scale was distributed to a sample composed of 346 professionals with an average age of 43 .

An exploratory factor analysis, a principal components analysis and an analysis of correlations between factors were applied. Such analyses have confirmed that the factorial structure of the scales converges towards the anticipated factors and it reaffirms its weight and confidence level. It can be concluded from this that the scale is valid for measuring the perception of teachers regarding the question being studied. The data indicates an optimum fit of the three-dimensional structure to the items of the scale. Keywords: scale; Likert; disruptive behaviour; school coexistence; education; validity; realiability; exploratory factor analysis.

\section{Introducción}

Los problemas de comportamiento en el aula se han convertido, desde hace tiempo, en uno de los temas más debatidos y analizados dentro del ámbito educativo (Albaladejo, Ferrer, Reig y Fernández, 2013; Armas, 2007; Barreiro, 2007; Díaz, Martínez y Martín, 2010; Moreno y Soler, 2006; Muñoz, Carreras y Braza, 2004; Orts, 2011; Urbina, Simón y Echeita, 2011). En este sentido, es importante señalar que este problema no solo se localiza en un área concreta, el aula, sino que parece tener una transcendencia que se manifiesta tanto en la escuela como en la sociedad en general. Una de las percepciones más extendidas entre el profesorado se refiere al alarmante aumento de las dificultades de convivencia de los centros educativos. La preocupación por la dinámica que se produce dentro de las aulas de diferentes niveles educativos se ha incrementado de forma exponencial y, como consecuencia de ello, el sistema se enfrenta actualmente con grandes obstáculos a la hora de regular la convivencia, combatiendo fenómenos ya conocidos y no tan novedosos, pero sí alarmantes por su actual visibilidad social

Una prueba clara de esta creciente preocupación se refleja en la creación en nuestro país, ya en el año 2007, del Observatorio Estatal de la Convivencia Escolar como órgano

* Dirección para correspondencia [Correspondence address]:

Marina Álvarez Hernández. Universidad de Oviedo, Facultad de Formación del Profesorado y Educación, Departamento de Psicología.C/ Aniceto Sela, s/n, 33005, Oviedo (España).

E-mail: amarina@uniovi.es colegiado de la Administración General del Estado (Ministerio de Educación y Ciencia, 2007), con el objetivo principal de contribuir a la construcción activa de un ambiente de convivencia escolar adecuado y también de diseñar estrategias y medidas para corregir y prevenir situaciones de violencia escolar. Asimismo, es significativo resaltar que los resultados de la última Encuesta Internacional sobre Enseñanza y Aprendizaje (Teaching and Learning International Survey, TALIS), elaborada por la OECD y publicada en junio de 2014, reflejan esta situación. Esta encuesta, que pretende servir de base para definir y revisar las políticas educativas con el objetivo de mejorar la calidad educativa, examina algunos indicadores vinculados con la educación, entre ellos, la valoración del clima escolar. Los resultados de este último informe TALIS revelan, de forma clara, que las conductas disruptivas constituyen una de las principales preocupaciones del profesorado español.

Numerosos estudios confirman estas percepciones (Busquets, Martín, Rosselló y Sáez, 2010; Simón, Gómez y Alonso, 2013; Urbina et al., 2011) y, concretamente, en el Principado de Asturias, un considerable número de profesores cita los problemas de comportamiento como uno de los obstáculos más graves para el desarrollo de la docencia tanto en educación primaria como en secundaria (Álvarez, Castro, Campo y Álvarez, 2005; Álvarez, Álvarez, Castro y Campo, 2008).

Es evidente que en los últimos años han aumentado las dificultades para impartir las clases debido a lo que se conoce como disrupción en el aula. Ello lo demuestra no solo la numerosa publicación de investigaciones en nuestro país en 
torno al tema (Foces, Marugán y Caño, 2002; Gotzens, Badía, Genovard y Dezcallar, 2010; Gotzens, Genovard, Badía y Castelló, 2003; Marchesi, 2005; Moreno y Soler, 2006; Saldaña, 2001, etc.), sino también la frecuencia con la que dicha cuestión aparece en los medios de comunicación.

En el año 2005, de acuerdo con el proyecto de la Ley Orgánica de Educación (LOE) sobre planes de convivencia como elementos básicos del proyecto Educativo del Centro, se establece un acuerdo básico entre el Ministerio de Educación y Ciencia y las Organizaciones Sindicales sobre condiciones sociolaborales del profesorado. En dicho acuerdo se propone establecer un plan de actuación para la promoción y mejora de la convivencia escolar y se incluyen una serie de compromisos de actuación encaminados a lograr dicho objetivo (Ministerio de Educación y Ciencia, 2005). El 23 de febrero de este mismo año, y como consecuencia del seguimiento de aquel acuerdo, se firma y publica el Real Decreto en el que se crea el Observatorio Estatal de la Convivencia Escolar (Ministerio de Educación y Ciencia, 2007), al que nos referíamos más arriba. Este Observatorio, en colaboración con las Comunidades Autónomas, ha permitido contar con un instrumento de autodiagnóstico de la convivencia escolar que permite, en distintos momentos, establecer comparativas con el objetivo de conocer la evolución de la convivencia en los centros.

Todo esto confirma que el problema de la disciplina en el aula es real y tiene su máxima actualidad; esta situación explica la proliferación de programas y planes que han surgido y que se vienen aplicando en muchos centros para abordar esta problemática. Las administraciones educativas han tomado clara conciencia de este hecho y han implantado nuevas medidas para intentar responder al problema. En el Principado de Asturias (Principado de Asturias, 2001a, 2001b) se publicaron unas Resoluciones de la Consejería de Educación y Cultura, por las que se aprobaban las instrucciones que regulan la organización y el funcionamiento de las Escuelas de Educación Infantil, de los Colegios de Educación Primaria y de los Institutos de Educación Secundaria. En ellas aparecía por primera vez la necesidad de que los centros tengan un programa de educación para la convivencia incluido en el Reglamento de Régimen Interior. Posteriormente, en el año 2006 se crea el Observatorio de la Infancia y de la Adolescencia del Principado de Asturias, dicho observatorio cuenta con un Foro de debate para la Convivencia entre iguales. Solo un año después, se publica otro decreto (Principado de Asturias, 2007), por el que se regulan los derechos y deberes del alumnado y normas de convivencia en los centros docentes no universitarios sostenidos con fondos públicos del Principado de Asturias. Y, muy recientemente, en el año 2013, se promulga una ley que refleja la preocupación social en torno a este tema (Ley del Principado de Asturias 3/2013, de 28 de junio, de medidas de autoridad del profesorado). Esta ley tiene como objetivo reforzar la autoridad de maestros y mejorar la convivencia en los centros educativos, asimismo pretende facilitar que los maestros puedan ejercer sus funciones en un clima adecuado, de orden, de disciplina y de respeto.

Esta preocupación en el Principado de Asturias se refleja también en la creación de programas dirigidos a intervenir en aspectos clave para la mejora de la convivencia escolar y la prevención de conflictos. Prueba de ello es el Programa EncuentrAstur por la Convivencia, cuyo objetivo es la formación conjunta de todos los miembros de la Comunicad Educativa en estrategias pacíficas de resolución de conflictos en los contextos. A este programa se une el de Mediación Escolar para la resolución de Conflictos, un programa más global de promoción de la participación y la convivencia escolares, que tiene como eje central el Plan Integral de Convivencia de los centros y que incluye todas las medidas encaminadas hacia la creación de una cultura de mejora de la convivencia.

Ciertamente, uno de los problemas actuales en la escuela es el de las conductas disruptivas. Cuando los comportamientos de los alumnos no encajan con los valores, motivaciones u objetivos del proceso educativo, a menudo surgen las conductas disruptivas en el aula (Fernández, 1999). Este fenómeno, junto con el absentismo, es uno de los problemas que más preocupan actualmente a los docentes (Simón et al., 2013; Urbina et al., 2011).

Tattum (1997) explica que las conductas disruptivas, en el lenguaje de los profesores, se interpretan como un conglomerado de conductas inapropiadas, como son: falta de cooperación y mala educación, insolencia, desobediencia, provocación y agresión, hostilidad y abuso, impertinencia, amenazas, etc. Dicha disrupción genera un escenario muy apropiado para no aprender y para dificultar la buena marcha de la clase.

El último informe realizado por el Observatorio Estatal de la Convivencia Escolar (Díaz, Martínez y Martín, 2010) señala la importancia de disponer de procedimientos que permitan evaluar la convivencia escolar desde una perspectiva integral. Por todo ello, las conductas disruptivas exigen un profundo análisis que ayude a encontrar sus causas y las posibles soluciones, que permita, en definitiva, elaborar y planificar estrategias de prevención y de solución. A pesar de la importancia de este problema, en España no se dispone de instrumentos de medida que evalúen de forma objetiva las opiniones del profesorado sobre las conductas disruptivas. En este contexto, el objetivo principal de este trabajo, consiste en desarrollar y validar una nueva escala con el propósito de conocer la opinión de los profesionales en relación a esta cuestión. Conseguida tal validación y, a partir de la aplicación de la escala, se podrán cumplir importantes objetivos en investigaciones futuras con el fin de formular propuestas novedosas para mejorar los problemas de comportamiento en el aula. Tras una revisión detallada, se ha podido comprobar que no existe ninguna escala de las mismas características que la presentada en este trabajo. Existen instrumentos que evalúan el clima social del centro (Haynes, Emmons y Comer, 1993; Trianes, Blanca, de la Morena, Infante y Raya, 2006) y otros que evalúan los comportamientos violentos en los centros educativos (Álvarez, Álvarez, González, Núñez y 
González, 2006; Avilés, 1999). Hay otras investigaciones recientes (Gotzens et al., 2010) que hacen un estudio comparativo entre tres muestras de docentes pertenecientes a distintas comunidades, y proporcionan una visión sobre la importancia que los docentes conceden a las conductas disruptivas y sobre cómo las diversas perspectivas y valoraciones afectan al quehacer diario del profesorado. Ninguno de estos estudios citados centra el problema, de forma específica, de las conductas disruptivas dentro del aula desde la perspectiva del profesorado.

El objetivo general de esta investigación consistirá, por lo tanto, en desarrollar y validar una nueva escala para conocer lo que opinan los profesionales de la educación en infantil, primaria y secundaria (centros específicos) sobre el aumento de las conductas disruptivas; los objetivos específicos de este estudio consistirán en recabar su opinión sobre posibles causas y sobre posibles medidas de mejora. La hipótesis de partida es que los ítems propuestos en la escala conformarán los factores que permitan analizar el aumento de las conductas disruptivas en las aulas.

\section{Método}

\section{Muestra}

La muestra (véase Tabla 1) está compuesta por 346 profesionales que trabajan o han trabajado en la atención al alumnado con necesidad específica de apoyo educativo (maestros tutores, maestros especialistas, orientadores, educadores...) del Principado de Asturias.

La recogida de datos se ha llevado a cabo durante el curso 2013-2014, en centros educativos ordinarios y específicos, financiados con fondos públicos y concertados.

Han participado profesionales de todos los centros específicos del Principado (28.3\%) y de los centros ordinarios $(71.7 \%)$. Se han agrupado en especialidades diferentes: maestros especialistas en educación especial $(24.6 \%)$, maestros de primaria $(21.4 \%)$, maestros de infantil $(18.8 \%)$, maestros de audición y lenguaje $(13.3 \%)$, orientadores $(8.1 \%)$, auxiliares técnicos educativos $(5.8 \%)$, profesores técnicos en servicios a la comunidad $(1.4 \%)$ y otras especialidades $(4.6 \%)$. Sus edades oscilan entre los 24 y los 68 años, siendo la media 43.47 años. De ellos, el $82.9 \%$ son mujeres y el $13.6 \%$ son hombres.

Los años de experiencia de los profesionales que han participado en centros ordinarios varían desde 0 hasta 42 y en centros específicos desde 0 hasta 33 años.

Tabla 1. Descripción de la muestra.

\begin{tabular}{|c|c|c|c|}
\hline & & $\mathbf{N}$ & $\%$ \\
\hline \multirow[t]{3}{*}{$\overline{\text { Género }}$} & Hombre & 47 & 13.6 \\
\hline & Mujer & 287 & 82.9 \\
\hline & No responde & 12 & 3.5 \\
\hline \multirow[t]{2}{*}{ Tipo de Centro Educativo } & Ordinarios & 248 & 71.7 \\
\hline & Específicos & 98 & 28.3 \\
\hline \multirow[t]{10}{*}{ Especialidad } & Maestro de Primaria & 74 & 21.4 \\
\hline & Maestro de Infantil & 65 & 18.8 \\
\hline & Educación Especial & 85 & 24.6 \\
\hline & Audición y Lenguaje & 46 & 13.3 \\
\hline & Profesor Técnico de servicios a la comunidad & 5 & 1.4 \\
\hline & Orientador & 28 & 8.1 \\
\hline & Auxiliar educador & 20 & 5.8 \\
\hline & Otras especialidades & 16 & 4.6 \\
\hline & Perdidos Sistema & 7 & 2.0 \\
\hline & Mínimo & Máximo & Media \\
\hline$\overline{\text { Edad }}$ & 24 & 68 & 43.47 \\
\hline Experiencia en centros específicos & 0 & 33 & 4.49 \\
\hline Experiencia en centros ordinarios & 0 & 42 & 13.87 \\
\hline
\end{tabular}

\section{Instrumento}

Escala de Conductas Disruptivas (ECD): el objetivo de la escala es evaluar la percepción del profesorado sobre las conductas disruptivas en educación infantil y primaria. En un principio se elaboró un cuestionario que constaba de 25 ítems cuya escala psicométrica utilizada fue el método de evaluaciones sumarias o escala Likert, con puntuación de 1 a
5, donde 1 equivale a "muy en desacuerdo" y 5 a "muy de acuerdo".

El primer paso llevado a cabo, consistió en realizar una exhaustiva revisión bibliográfica sobre el tema objeto de estudio. En este proceso cabe destacar que no existen instrumentos específicos que analicen la percepción del profesorado respecto a este tipo de conductas. Tal y como se ha comentado en la introducción, existen otros instrumentos, pero que no centran el problema, de forma específica, de las con- 
ductas disruptivas dentro del aula desde la perspectiva del profesorado (Álvarez et al., 2006; Avilés, 1999; Gotzens et al., 2010; Haynes et al., 1993; Trianes et al., 2006).

Como consecuencia, en segundo lugar se ha procedido a diseñar un cuestionario propio basado en estudios previos sobre la percepción del profesorado sobre el alumnado con necesidades específicas de apoyo educativo (Álvarez et al., 2005; Álvarez et al., 2008; Álvarez, Campo, Castro y Álvarez, 2009 y Campo, Castro, Álvarez, Álvarez y Torres, 2010).

En la tercera fase se procedió a validar la ECD en cuanto a contenido (validez de contenido), para ello se sometió a una valoración por parte de expertos. Se contactó con quince profesionales especialistas en el campo de la educación, entre los que había inspectores de educación, maestros tutores de primaria, maestros especialistas en pedagogía terapéutica, directores y orientadores de centros educativos ordinarios y específicos, técnicos especialistas del Servicio de Alumnado, Orientación y Participación Educativa de la Consejería de Educación, Cultura y Deporte del Principado y profesores universitarios expertos en diseño de pruebas de valoración, todos ellos con una dilatada experiencia. Su valoración consistió en evaluar de 1 a 10, la pertinencia de cada uno de los ítems, así como la claridad de los mismos, su orden y estructura. Tras la evaluación de estos expertos, se procedió, en primer lugar, a la discusión de aportaciones y sugerencias y, posteriormente, a realizar las modificaciones propuestas, eliminando algunos ítems que habían recibido una valoración baja, modificando la redacción de otros ítems, y añadiendo algún ítem que podía ofrecer información muy relevante para el Servicio de Alumnado, Orientación y Participación Educativa del Principado. De este modo, la ECD quedó formada por 21 ítems específicos.

Por último, se procedió a la aplicación de la escala a la muestra seleccionada.

\section{Procedimiento}

En la selección de la muestra se tuvieron en cuenta cuarenta y dos centros educativos del Principado de Asturias, diez centros específicos y treinta y dos ordinarios. Se solicitó la colaboración de los centros mediante llamada telefónica y, una vez confirmada la participación, se elaboró la lista de centros participantes. En el momento de la entrega de cada escala, se procedió a explicar a los profesionales implicados el objetivo de la investigación, incidiendo en la importancia que tenía la cumplimentación de todos los ítems. La recogida se efectuó en persona, agradeciendo el alto nivel de participación de los profesionales.

\section{Análisis de datos}

Siguiendo las recomendaciones de Lloret, Ferreres, Hernández y Tomás (2014), que aconsejan hacer un uso secuencial del AFE y el AFC, se ha optado por realizar un primer AFE para identificar el número y composición de los factores comunes (variables latentes) necesarios para explicar la varianza común del conjunto de ítems analizado y de esta manera validar la escala.

Se calcularon los estadísticos univariados para cada ítem (media y desviación típica). Para analizar la dimensionalidad de la escala se llevó a cabo un análisis factorial exploratorio (AFE). En la determinación del número de factores se utilizó el método de Implementación Óptima de Análisis Paralelos propuesto por Timmerman y Lorenzo (2011) llevándose a cabo 10.000 remuestreos. El AF se realizó a partir de las correlaciones policóricas entre los ítems. El método de factorización fue el de mínimos cuadrados no ponderados y se utilizó el método Promin de rotación (Ferrando y Lorenzo, 2014). Para cada dimensión, de forma independiente, se calculó la correlación parcial ítem-test para la estimación del índice de discriminación de los ítems. Para el cálculo de la fiabilidad se utilizó el coeficiente alfa de Cronbach para datos ordinales (Elosua y Zumbo, 2008).

\section{Resultados}

Una vez realizado el AFE se rotan tres factores con 15 ítems y se han eliminado 6 por tener una carga inferior a .30. El estadístico de Bartlett [1800.3 ( $\mathrm{df}=105 ; \mathrm{P}<.001)$ ], así como el test de Kaiser-Meyer-Olkin $(\mathrm{KMO})=.75$, indican una buena adecuación de los datos para ser sometidos a análisis factorial. Los tres factores extraídos explican el $56 \%$ de la varianza total, el índice de bondad de ajuste (GFI) fue de .98 y la raíz cuadrática media de los residuales (RMSR) de 0.054. Todos estos datos indican un muy buen ajuste de la estructura tridimensional para estos ítems (García, Gayo y Miranda, 1998). (Véase Tabla 2)

El primer factor, denominado "propuestas de mejora docente", agrupa un conjunto de ítems $(9,10,11,12,13$ y 14) que recogen propuestas para mejorar la docencia en el aula. Numerosos estudios avalan la eficacia de diversas estrategias para el manejo de la convivencia en el aula (Sánchez, Ribas y Trianes, 2006). Todos ellos apuestan por la utilización de estrategias de autogestión, de flexibilidad y que promuevan conductas menos directivas dentro del aula (Martín, Fernández, Andrés, Barrio y Echeita, 2003; Palomero y Fernández, 2001; Trianes y Fernández, 2001).

El segundo factor, denominado "modalidades de escolarización”, englobaría los ítems 7, 8 y 15. Numerosas investigaciones han sido desarrolladas en torno a las modalidades de escolarización (Echeita y Calderón, 2014) y en torno a los tipos de agrupamiento como variables relevantes para la trayectoria educativa de los alumnos (Pulido, Martín y Lucas, 2011).

El tercer factor, denominado "opinión sobre las causas del incremento de problemas en el aula", agrupa los ítems 1, $2,3,4,5$ y 6 . Como muestran numerosas investigaciones, los cambios producidos en la sociedad repercuten en los límites y normas impuestas en el alumnado (Garrido, 2005; Unceta, 2008). Asimismo, parece probado que la ausencia de límites y normas está directamente relacionada con la implicación familiar (Barrero, 2010; Medrano, 2005; Ordóñez, 2006). 
Además es importante señalar que se han publicado numerosos estudios que tratan de analizar cómo la proliferación de las redes sociales y del uso de dispositivos móviles puede influir en las conductas de los alumnos (Carbonell, Fúster, Chamarro y Oberst, 2012; Castellana, Sánchez, Graner y Beranuy, 2007; Echeburúa y De Corral, 2012; Levis, 2002; López y Sabater, 2014; Muñoz y Agustín, 2005).

Los coeficientes de fiabilidad estimados fueron de $.90 \mathrm{pa}-$ ra el primer factor, .78 para el segundo y .93 para el tercero (véase tabla 2).

En la misma tabla 2 se recogen los estadísticos principales de los ítems que conforman la escala (media, desviación típica). Se puede observar que las medias obtenidas en algunos de los ítems son bastante altas, en concreto los ítems correspondientes al factor 1, "propuestas de mejora docente", entre los que destaca la puntuación más alta del ítem "mejorar la coordinación entre los diferentes servicios" (4.36). También llama la atención la media tan elevada (4.25) que obtiene el ítem "falta de normas y límites en el entorno familiar" perteneciente al factor 3 ("opinión sobre las causas del incremento de problemas en el aula").

Cabe destacar que existen tres ítems, todos ellos pertenecientes al factor 2 ("modalidades de escolarización"), cuya puntuación está por debajo del valor central, este factor hace referencia a modalidades de escolarización segregada para este tipo de alumnado. En esta misma situación se encuentra el ítem "falta de normas y límites en el entorno escolar" (2.66), perteneciente al factor 3 ("opinión sobre las causas del incremento de problemas en el aula"). En la tabla 3 se muestran las correlaciones entre los factores.

Tabla 2. Media, desviación típica, índice de discriminación, matriz factorial rotada y análisis de fiabilidad de variables y factores

\begin{tabular}{|c|c|c|c|c|c|c|}
\hline & $M$ & $S D$ & $I D$ & F1 & F2 & F3 \\
\hline \multicolumn{7}{|l|}{ Considera que el incremento de los problemas de conducta es debido a: } \\
\hline V1. Un cambio general en la sociedad & 3.85 & .929 & .357 & 0.246 & 0.039 & 0.319 \\
\hline V2. La falta de normas y límites en el entorno escolar & 2.66 & 1.186 & .234 & 0.130 & 0.068 & 0.160 \\
\hline V3. La falta de normas y límites en el entorno familiar & 4.25 & .837 & .364 & 0.149 & 0.110 & 0.316 \\
\hline V4. El uso/abuso de las redes sociales & 3.23 & 1.025 & .584 & -0.066 & -0.045 & 0.961 \\
\hline V5. El abuso de las aplicaciones móviles & 3.12 & 1.028 & .556 & -0.094 & -0.043 & 0.920 \\
\hline V6. Falta de coordinación entre familia y escuela & 3.47 & 1.074 & .396 & 0.253 & 0.089 & 0.316 \\
\hline $\begin{array}{l}\text { Para dar una respuesta educativa adecuada a las necesidades del alumnado con } \\
\text { problemas de conducta sería conveniente: }\end{array}$ & $M$ & $S D$ & ID & F1 & F2 & F3 \\
\hline V7. Crear aulas específicas en centros ordinarios & 2.60 & 1.335 & .411 & 0.028 & 0.629 & 0.009 \\
\hline V8. Crear aulas específicas en centros específicos & 2.59 & 1.248 & .521 & -0.093 & 0.833 & -0.021 \\
\hline V9. Crear programas específicos adaptados & 4.21 & .825 & .563 & 0.569 & 0.132 & 0.027 \\
\hline V10. Modificar la metodología del aula docente & 3.86 & 1.032 & .676 & 0.787 & -0.090 & -0.014 \\
\hline V11. Flexibilizar la organización del aula & 4.09 & .858 & .729 & 0.817 & -0.048 & 0.007 \\
\hline V12. Mejorar la formación del profesorado & 4.23 & .943 & .627 & 0.778 & -0.072 & -0.053 \\
\hline V13. Mejorar la coordinación entre los diferentes servicios & 4.36 & .805 & .646 & 0.789 & 0.038 & 0.022 \\
\hline V14. Mejorar la coordinación entre los profesionales del centı & 4.18 & .901 & .641 & 0.759 & 0.009 & 0.015 \\
\hline V15. Escolarizarlos en la modalidad de escolarización combinada & 2.65 & 1.187 & .349 & 0.011 & 0.471 & 0.017 \\
\hline Coeficiente de fiabilidad $(\alpha)$ & & & & .90 & .78 & .93 \\
\hline
\end{tabular}

Tabla 3. Matriz de correlaciones entre los factores.

\begin{tabular}{llcccc}
\hline Factor & F 1 & F 2 & F 3 \\
\hline F 1 & - & & & \\
F & 2 & -0.097 & - & & \\
F 3 & 0.240 & 0.034 & - \\
\hline
\end{tabular}

\section{Discusión y conclusiones}

Atendiendo a los objetivos a corto plazo planteados en este artículo, se puede afirmar que tanto los resultados cualitativos como los cuantitativos aportan sugerencias para la versión final de la escala y se confirma la hipótesis de partida, los ítems propuestos conforman los factores que permiten analizar el aumento de las conductas disruptivas en las aulas.

Desde el punto de vista cualitativo, los valiosos aportes de los expertos permitieron realizar modificaciones propuestas, eliminando, como hemos dicho anteriormente, algunos ítems que habían recibido una valoración baja, modificando la redacción de algunos ítems, y añadiendo algún ítem que podía ofrecer información relevante.
Desde el punto de vista cuantitativo, se comprueba que la estructura factorial de las escalas converge con los factores previstos y reafirma su peso y el nivel de confianza.

La Tabla 2 muestra la agrupación de los ítems por factores estimados.

El primer factor (Tabla 2), "propuestas de mejora docente", agrupa un conjunto de ítems que recogen propuestas para mejorar la docencia en el aula. Los ítems agrupados han sido los siguientes: crear programas específicos adaptados, modificar la metodología del aula docente, flexibilizar la organización del aula, mejorar la formación del profesorado, mejorar la coordinación entre los diferentes servicios y mejorar la coordinación entre los profesionales del centro que trabajan con el alumno.

Existen numerosos estudios que avalan la eficacia de diferentes estrategias en el manejo de la convivencia en el aula (Sánchez, Rivas y Trianes, 2006). La mayoría de dichos estudios coinciden en la utilización de medidas que promocionen comportamientos menos directivos dentro del aula, que trabajen la autogestión en el establecimiento de normas así co- 
mo la autonomía del alumnado, la flexibilidad organizativa, etc. (Martín et al., 2003; Palomero y Fernández, 2001; Trianes y Fernández, 2001).

En el año 2005 se presentó un programa para la mejora de la convivencia que presenta resultados positivos (Sánchez, 2005; Trianes, Cardelle, Blanca y Muñoz, 2003). Dicho programa supone un buen instrumento para el profesorado y demuestra que una vía es la prevención, el trabajo antes de que las conductas disruptivas sean graves. En el mismo se proponen medidas como mejorar las relaciones interpersonales, lo que implicaría la mejora de la coordinación; insistir en el desarrollo profesional, lo que supondría la mejora de la formación docente; y evitar la directividad, lo que flexibilizaría la organización. Todas estas medidas se recogen en los ítems que agrupan el factor 1.

El segundo factor, "modalidades de escolarización" (Tabla 2), engloba los siguientes ítems: crear aulas específicas en centros ordinarios, crear aulas específicas en centros específicos y escolarizar en modalidad combinada.

En la actualidad no hay duda de que todos los agentes educativos defienden la educación inclusiva (Bottrell y Goodwin, 2011; Parrilla, Muñoz y Sierra, 2013). Parafraseando a Pilar Arnaiz: "La filosofía de la inclusión defiende una educación eficaz para todos, sustentada en que los centros, en tanto comunidades educativas, deben satisfacer las necesidades de todos los alumnos, sean cuales fueren sus características personales, psicológicas o sociales (con independencia de si tienen o no discapacidad)", (Arnaiz, 2011, p. 11). Sin embargo, no es difícil entender que avanzar hacia una educación inclusiva supone un reto de enorme envergadura y complejidad (López, Echeita y Martín, 2010). Son numerosas las ocasiones en que se nos presentan dilemas difíciles de resolver. Por ejemplo, determinadas formas de escolarizar al alumnado pueden ser técnicamente más efectivas que otras, pero pueden conllevar discriminación y exclusión de algunos grupos (Dyson y Millward, 2000). G. Echeita, en el año 2007, publica un artículo en la Revista Latinoamericana de Educación Inclusiva cuyo título recoge de forma muy manifiesta la idea que tratamos de desarrollar: "Del dicho al hecho hay un trecho". En dicho artículo se reflexiona sobre la distancia entre nuestros valores e intenciones declaradas hacia la inclusión educativa y social de los más vulnerables y las políticas y prácticas educativas que deberían sustentarla (Echeita, 2007). Existen publicaciones que evidencian este hecho. Entre los años 2005 y 2010 se llevaron a cabo, en el Principado de Asturias, tres investigaciones que demuestran cómo los maestros de primaria, los profesores de secundaria y los especialistas en pedagogía terapéutica opinan que el colectivo de estudiantes con problemas de comportamiento, junto con el perteneciente a minorías étnicas, son percibidos como los menos aceptados por los compañeros, los que menor participación tienen en las actividades y los que ofrecen mayores dificultades al centro y a la labor docente. Por ello, los profesionales apuestan por otras modalidades de escolarización diferentes al aula ordinaria. (Álvarez et al., 2005; Álvarez et al, 2008; Campo et al., 2010). En dichas investigaciones los da- tos muestran que existe cierta confusión con respecto a la escolarización del alumnado con problemas del comportamiento.

El tercer factor, "Opinión sobre las causas del incremento de problemas en el aula" (Tabla 2) agrupa los siguientes ítems, a saber: un cambio general en la sociedad, la falta de normas y límites en el entrono escolar, la falta de normas y límites en el entorno familiar, el uso/abuso de las redes sociales, el abuso de las aplicaciones móviles y la falta de coordinación entre escuela y familia.

Es algo probado que en las últimas décadas se han producido cambios en la sociedad que afectan a los límites y a las normas impuestas a nuestros jóvenes (Echevarría, 2002; Orts, 201; Torrego, 2004). Efectivamente, como numerosas investigaciones confirman, los cambios sociales tienen una proyección inmediata en las conductas del alumnado (Unceta, 2008). La bibliografía consultada apunta a que uno de los factores desencadenantes de los problemas de comportamiento en las aulas tiene que ver con estos cambios sociales que llevan implícitos reducción de normas y de límites. Por poner un ejemplo, el desarrollo de la sociedad actual ha conllevado mayores comodidades en el estilo de vida, retraso en la adopción de roles de responsabilidad, desatención en la formación de la conciencia, del código moral, etc. (Garrido, 2005).

En sintonía con lo expuesto, cabe decir que la reducción o incluso la ausencia de límites y de normas, tanto en la escuela como en la familia, están directamente relacionados con los cambios sociales. Son abundantes las publicaciones que abordan el tema de la familia y la escuela y la falta de sintonía entre ambas, tanto en lo que respecta a normas, como a expectativas, a modelos de referencia, etc. (Barrero, 2010; Medrano, 2005; Mosquera y Aguirre, 1996). La familia es un sistema social que, junto con otros contextos, ejerce una influencia de prioridad en el desarrollo de valores de las personas (Medrano, 2005). Es prioritario que familia y escuela compartan la responsabilidad de educar y que lo hagan de una forma coherente y coordinada, evitando las discrepancias y los antagonismos (Ordóñez, 2006).

Por último, respecto a este tercer factor, en los últimos años se han publicado investigaciones que tratan de analizar cómo puede estar influyendo la proliferación de las redes sociales y el uso de los dispositivos móviles en comportamientos y actitudes (Carbonell et al., 2012; Castellana et al., 2007; Echeburúa y De Corral, 2012; Levis, 2002; López y Sabater, 2014; Muñoz y Agustín, 2005). En todos estos estudios se sostiene una idea clave, a saber, que en los últimos años, está emergiendo un nuevo tipo de desajustes comportamentales producto de la generalización de las TIC (Castellana et al., 2007). Parece que el propio diseño de estas herramientas puede afectar a la voluntad de control (Saldaña, 2001).

$\mathrm{Al}$ iniciar este proceso metodológico nos proponíamos obtener un instrumento de recogida de información, fiable y válido, para conocer y analizar las percepciones que los profesionales poseen sobre el aumento de las conductas disruptivas en las aulas. Finalmente podemos concluir que, a la luz 
de lo comentado en el apartado "Resultados", los datos indican un óptimo ajuste de la estructura tridimensional para los ítems que componen la escala. Consideramos que esta in-

\section{Referencias}

Albaladejo, N., Ferrer, R., Reig, A. y Fernández, M.D. (2013). ¿Existe violencia escolar en educación infantil y primaria? Una propuesta para su evaluación y gestión. Anales de psicología, 29(3), 1060-1069. doi: http://dx.doi.org/10.6018/analesps.29.3.158431

Álvarez, L., Álvarez, D., González, P., Núñez, J.C. y González, J.A. (2006). Evaluación de los comportamientos violentos en los centros educativos. Psicothema, 18(4) 686-695.

Armas, M. (2007). Prevención e intervención ante problemas de conducta. Estrategias para centros educativos y familias. Madrid: Wolters Kluwer España, S.A.

Arnaiz, P. (2003). Educación inclusiva: una escuela para todos. Málaga: Editorial Aljibe.

Arnaiz, P. (2011). Luchando contra la exclusión: buenas prácticas y éxito escolar. Revista de Innovación Educativa, 21, pp. 23-35. doi:10.15304/ie.21.22

Álvarez Hernández, M.; Castro Pañeda, P.; Campo Mon, M.A. y Álvarez Martino, E. (2005). Actitudes de los maestros ante las necesidades educativas específicas. Psicothema, 17(4) 601-606.

Álvarez Martino, E.; Álvarez Hernández, M.; Castro Pañeda, P. y Campo Mon, M.A. (2008). Funcionamiento de la integración en la Enseñanaza Secundaria Obligatoria según la percepción del profesorado. Psicothema, 20(1) 56-61.

Álvarez Martino, E.; Campo Mon, M.A.; Castro Pañeda, P. y Álvarez Hernández, M. (2009). Visión de los especialistas en pedagogía terapéutica de la integración del alumno inmigrante. Aula Abierta, 37, 57-66.

Avilés, J.M. (1999). CIMEI. Cuestionario sobre intimidación y maltrato entre iguales. Valladolid: Autor.

Barreiro, T. (2007). Conflictos en el aula. Buenos Aires: Centro de Publicaciones Educativas y Material Didáctico.

Barrero, R. L. (2010). Familia vs. Escuela. Pedagogía Magna, (5), 154-159.

Beranuy, M., Chamarro, A., Graner, C. y Sánchez, X. (2009). Validación de dos escalas breves para evaluar la adicción a Internet y el abuso de móvil. Psicothema, 21(3), 480-485

Bottrell, D. y Goodwin, S. (Eds.) (2011). Schools, Communities and Social Inclusion. South Yarra. Australia: Palgrave MacMillan.

Busquets, C. G., Martín, M. B., Rosselló, C. G. y Sáez, T. D. (2010). Estudio comparativo de la gravedad atribuida a las conductas disruptivas en el aula. Electronic Journal of Research in Educational Psychology, 8(20), 33-58.

Campo Mon, M.A.; Castro Pañeda, P.; Álvarez Martino, E.; Álvarez Hernández, M. y Torres Manzanera, C. (2010). Funcionamiento de la integración según la percepción de los maestros especialistas en Pedagogía Terapéutica. Psicothema, 22(4) 797-805.

Carbonell, X., Fúster, H., Chamarro, A. y Oberst, U. (2012). Adicción a internet y móvil: una revisión de estudios empíricos españoles. Papeles del Psicólogo, 33(2), 82-89.

Castellana, M., Sánchez, X., Graner, C. y Beranuy, M. (2007). El adolescente ante las tecnologías de la información y la comunicación: internet, móvil y videojuegos. Papeles del Psicólogo, 28(3), 196-204.

Cronbach, L.J. (1951). Coefficient alpha and the internal structure of test. Psichometrica, 16, 297-334. doi: 10.1007/BF02310555

Díaz, M. J., Martínez, R. y Martín, J. (2010). Estudio estatal de la convivencia escolar en la Educación Secundaria Obligatoria. Madrid: Ministerio de Educación.

Dyson, A. y Millward A. (2000). Schools and special needs: Issues of innovation and inclusion. London: Paul Chapman. DOI: http://dx.doi.org/10.4135/9781446219423

Echeburúa, E. y De Corral P. (2012) Adicción a las nuevas tecnologías y a las redes sociales en jóvenes: un nuevo reto. Adicciones, 22, 91-96.

Echeita, G. (2007). Del dicho al hecho hay un trecho. Revista Latinoamericana de Educación Inclusiva, 1(1), 29-36.

Echeita, G. y Calderón, I. (2014). Obstáculos a la inclusión: cuestionando concepciones y prácticas sobre la evaluación psicopedagógica. Ambits de Psicopedagogia i Orientació, 41 formación y su análisis generarán y modificarán, si se diese el caso, actuaciones para el futuro.

Echeverría, J. (2002). Ciencia y valores. Barcelona: Destino.

Elosua, P. y Zumbo, B. (2008). Coeficientes de fiabilidad para escalas de respuesta categórica ordenada. Psicothema 20(4), 896-901

Fernández, I. (1999). Prevención de la violencia y resolución de conflictos. Madrid: Narcea

Ferrando, P. J. y Lorenzo, U. (2014). El análisis factorial exploratorio de los ítems: algunas consideraciones adicionales. Anales de Psicología 30(3), 1170-1175. doi: http://dx.doi.org/10.6018/analesps.30.3.199361

Foces, J., Marugán, M. y Caño, M. (2002). Análisis y tratamiento de la conflictividad en un centro de educación secundaria. Revista Electrónica Interuniversitaria de Formación del Profesorado, 5(1), 1-5.

García, E., Gallo, P. y Miranda, R. (1998). Bondad de ajuste en el análisis factorial confirmatorio. Psicothema, 10(3), 717-724.

Garrido, V. (2005). Los hijos tiranos. El sindrome del emperador. Ariel: Barcelona.

Gotzens, C., Badía, C., Genovard, C. y Dezcallar, M.T. (2010). Estudio comparativo de la gravedad atribuida a las conductas disruptivas en el aula. Electronic Journal of Research in Educational Psychology, 8(20), 33-58.

Gotzens, C., Genovard, C., Badía, M. y Castelló, A. (2003). Percepciones de profesores y alumnos de E.S.O. sobre la disciplina en el aula. Psicothema, 15(3), 362-368.

Haynes, N. M., Emmons, C.L. y Ben, M. (1997). School climate as a factor in student adjustment and achievement. Journal of Educational and Psychological Consultation, 8, 321-339. doi: 10.1207/s1532768xjepc0803_4

Haynes, N. M., Emmons, C.L. y Comer, J.P. (1993). Elementary and middle school climate survey. New Haven, CT: Yale University Child Study Center.

Hofman, R. (1995). Establishing factor validity using variable reduction in confirmatory factor analysis. Educational and Psychological Measurement, 55, 572-582. doi: 10.1177/0013164495055004005

Hogan, T. P. (2004). Pruebas psicológicas. México: Manual Moderno.

Jiménez, M. (2012). Actuaciones socio-comunitarias y educativas inclusivas con alumnado en riesgo de exclusión social. Revista de Investigación en Educación, 10 (2), 2012, pp. 62-78.

Levis, D. (2002). Videojuegos: cambios y permanencias. Comunicación y Pedagogía, 184, 65-69.

Ley del Principado de Asturias 3/2013, de 28 de junio, de medidas de autoridad del profesorado. Boletín Oficial del Principado de Asturias, 154, de 4 de julio de 2013.

LOE, 2006. Ley Orgánica 2/2006, de 3 de mayo, de Educación. B.O.E. 4/05/2006.

LOMCE, 2013, Ley Orgánica /2013, de 9 de diciembre, para la mejora de la calidad de la educación. B.O.E. 10/12/2013.

López, L. y Sabater, C. (2014). Medios audiovisuales y acoso escolar: buenas prácticas para la prevención y promoción de la convivencia. Revista de Investigación en Educación, $\mathrm{n}^{\circ} 12$ (2), 2014, pp. 145-163.

López, M., Echeita, G. y Martín, E. (2010). Dilemas en los procesos de inclusión: explorando instrumentos para una comprensión de las concepciones educativas del profesorado. Revista Latinoamericana de Educación Inclusiva, 4(2), 155-176.

Lloret, S., Ferreres, A., Hernánez, A. y Tomás, I. (2014). El análisis factorial exploratorio de los ítems: una guía práctica, revisada y actualizada. Anales de psicología, 30(3), 1151-1169. http:/ /dx.doi.org/10.6018/analesps.30.3.199361

Marchesi, A. (2005). Controversias en la educación española. Madrid: Alianza Editorial.

Martín, E., Fernández, I., Andrés, S., Barrio del, C. y Echeita, G. (2003). Intervention to improve coexistence at school: Models and domains. Infancia y Aprendizaje: Journal for the study of Education and Development, 26(1), 75-95. doi: 10.1174/02103700360536446

Medrano, C. (2005). La familia como contexto básico del desarrollo de va- 
lores. Revista de Psicología General y Aplicada, 58(2), 239-263.

Moreno, A. y Soler, M. P. (Coords.). (2006). La disrupción en las aulas: problemas y soluciones. Madrid: Ministerio de Educación y Ciencia.

Mosquera, S. y Aguirre, E. (1996). Familia y escuela: una responsabilidad compartida. Rev. IN-FAN-CIA, 19-22.

Muñiz, J. (1997). Introducción a la teoría de respuesta de los items. Madrid: Pirámi-

Muñiz, J. (2003). Teoría clásica de los Tests. Madrid: Pirámide.

Muñoz, J.M.; Carreras, M. R. y Braza, P. (2004). Aproximación al estudio de las actitudes y estrategias de pensamiento social y su relación con los comportamientos disruptivos en el aula en la educación secundaria. Anales de psicología, 20(1), 81-91.

Muñoz, J. y Agustín, S. (2005). La adicción al teléfono móvil. Psicología conductual, 13(3), 481-493.

OECD (2013). New Insights from TALIS 2013. Teaching and Learning in Primar and Upper Secondary Education. OECD Publishing. http://dx.doi.org/10.1787/9789264226319-en

Ordóñez, R. (2006). Colaboración Familia-Escuela: Estrategias para Mejorar Dicha Cooperación. En R. Ordóñez y C. Hervás (Coords.), La Programación Didáctica en Educación Infantil: el Diseño de Unidades Didácticas, pp. 1 20. España. Aprende-Iea.

Orts, J. V. (2003). Resolución de conflictos en el aula. Madrid: Santillana

Orts, J. V. (2011). Cómo dar clase a los que no quieren. Barcelona: Grao.

Palomero, J.E. y Fernández, M. R. (2001). La violencia escolar, un punto de vista global. Revista Interuniversitaria de Formación de Profesorado, 41, 19-38.

Parrilla, A., Muñoz, Ma A. y Sierra, S. (2013). Proyectos Educativos con vocación comunitaria. Revista de Investigación en Educación, 11 (3), 2013, 15 31

Principado de Asturias (2013). Ley del Principado de Asturias 3/2013, de 28 de junio, de medidas de autoridad del profesorado. BOP A, 4-VII-2013.

Principado de Asturias (2001a). Resolución de 6 de agosto de 2001, de la Consejería de Educación y Cultura, por la que se aprueban las instrucciones que regulan la organización y funcionamiento de los Institutos de Educación Secundaria del Principado de Asturias, BOP A, 188, lunes 13 de agosto de 2001.

Principado de Asturias (2001b). Resolución de 6 de agosto de 2001, de la Consejería de Educación y Cultura, por la que se aprueban las instrucciones que regulan la organización y el funcionamiento de las Escuelas de Educación Infantil y de los Colegios de Educación Primaria del Principado de Asturias. BOPA, 188, lunes 13 de agosto de 2001.

Principado de Asturias (2006). Decreto 10/2006, de 24 de enero, del Observatorio de la Infancia y la Adolescencia del Principado de Asturias. BOP A, 7-II-2006.

Principado de Asturias (2007). Decreto 249/2007, de 26 de septiembre, por el que se regulan los derechos y deberes del alumnado y normas de convivencia en los centros docentes no universitario sostenidos con fondos públicos del principado de Asturias. BOPA, 22-X-2007.
Pulido, R.; Martín, G. y Lucas, B. (2011). La modalidad de agrupamiento educativo como variable relevante en el análisis de la violencia escolar. Revista de Educación, 356, 457-481. DOI: 10-4438/1988-592X-RE-2010356-047

Ruiz, M., Pino, M.J. y Herruzo, J. (2012). Revisión de la técnica "El juego del buen comportamiento". Análisis y Modificación de Conducta 32(144), 554-574.

Saldaña, D. (Coord.) (2001). Detección y prevención en el aula de los problemas del adolescente. España: Pirámide

Saldaña, D. (2001). Nuevas tecnologías: nuevos instrumentos y nuevos espacios para la psicología. Apuntes de Psicología, 19(1), 5-10.

Sánchez, A. M. (2005). Una intervención psicoeducativa sobre competencia social en alumnado de 9 a 15 años. Su evaluación con un diseño longitudinal. Tesis doctoral. Universidad de Málaga.

Sánchez, A. M., Rivas, M. T. y Trianes, M.V. (2006). Eficacia de un programa de intervención para la mejora del clima escolar: algunos resultados. Revista Electrónica de Investigación Psicoeducativa, 4(2), 353-370.

Simón, C., Gómez, P. y Alonso, J. (2013). Prevención de la disrupción en el aula: papel del clima motivacional de clase y de las estrategias de afrontamiento. Cultura y Educación, 25(1), 49-64. doi:10.1174/113564013806309037

Tattum, D. (1997). A whole-school response: from crisis management to prevention. The Irish Journal of Psychology, 18(2), 221-232. doi:10.1080/03033910.1997.10558141

Timmerman, M. E. y Lorenzo, U. (2011). Dimensionality assessment of ordered polytomous items with parallel analysis. Psychological Methods, 16(2), 2019-220. doi: 10.1037/a0023353

Torrego, J. C. (2004). Modelo integrado de regulación de la convivencia y tratamiento de conflictos: un proyecto que se desarrolla en centros de la comunidad de Madrid. Tabanque, 18(2004), 31-48.

Trianes, M. V. y Fernández, C. (2001). Aprender a ser persona y a convivir: un programa para secundaria. Bilbao: Descleé de Bower.

Trianes, M. V., Cardelle, M., Blanca, M. J. y Muñoz, A. (2003). Contexto social, género y competencia social autoevaluada en alumnos andaluces de 11-12 años. Revista Electrónica de Investigación Psicoeducativa y Psicopedagógica, 2(1), 38-55.

Trianes, Mª V., Blanca, M. J., de la Morena, L., Infante, L. y Raya, S.(2006). Un cuestionario para evaluar el clima social del centro escolar. Psicothema, 18 (2), 272-277

Unceta, A. (2008). Cambios sociales y educación notas para el debate. Revis ta de Educación, 347. 419-43

Urbina, C., Simón, C. y Echeita, G. (2011). Concepciones de los profesores acerca de las conductas disruptivas: análisis a partir de un marco inclusivo. Infancia y aprendizaje: Journal for the Study of Education and Development, 34(2), 205-217. doi: 10.1174/021037011795377584

(Articulo recibido: 17-03-2015; revisado: 21-10-2015; aceptado: 05-11-2015) 